LA-UR- $97-2857$

CONF-970707_- Title: $\mid \begin{aligned} & \text { SHEAR BAND FORMATION IN PLASTIC } \\ & \text { BONDED EXPLOSIVES (PBX) }\end{aligned}$

Author(s): $\quad$ T. N. Dey

J. N. Johnson

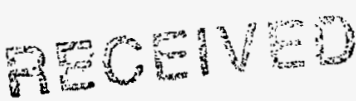

Nov 03 sog?

(1)

Submitted to: Shock Compression in Condensed Matter

19980219108

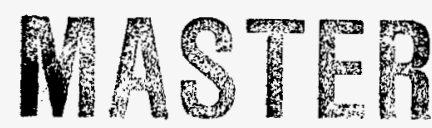

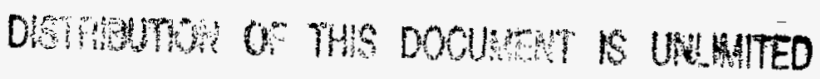
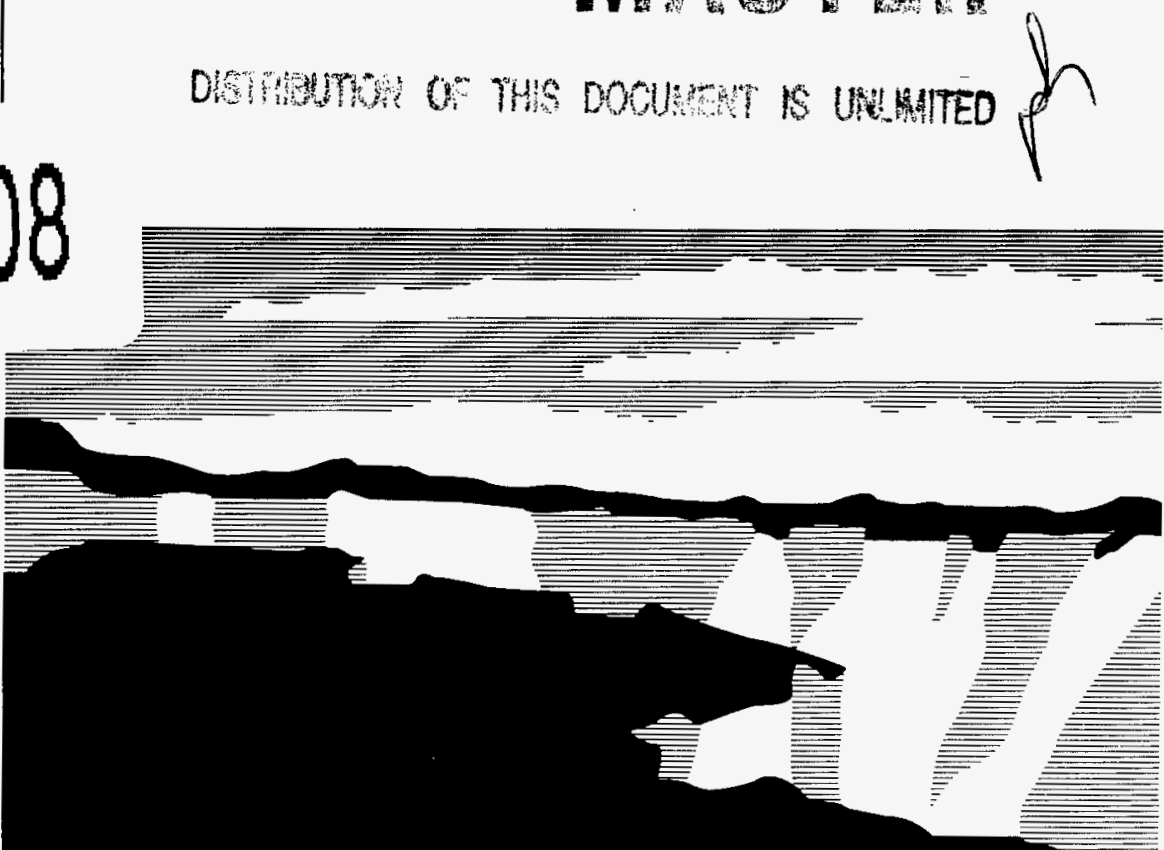

Los Alamos National Laboratory, an affirmative action/equal opportunity empldyer, is operated by the University of California for the U.S. Department of Energy under contract W-7405-ENG-36. By acceptance of this article, the publisher recognizes that the U.S. Government retains a nonexclusive, royalty-free license to publish or reproduce the published form of this contribution, or to allow others to do so, for U.S. Government purposes. The Los Alamos National Laboratory requests that the publisher identify this article as work performed under the auspices of the U.S. Department of Energy. 


\section{DISCLAIMER}

This report was prepared as an account of work sponsored by an agency of the United States Government. Neither the United States Government nor any agency thereof, nor any of their employees, makes any warranty, express or implied, or assumes any legal liability or responsibility for the accuracy, completeness, or usefulness of any information, apparatus, product, or process disclosed, or represents that its use would not infringe privately owned rights. Reference herein to any specific commercial product, process, or service by trade name, trademark, manufacturer, or otherwise does not necessarily constitute or imply its endorsement, recommendation, or favoring by the United States Government or any agency thereof. The views and opinions of authors expressed herein do not necessarily state or reflect those of the United States Government or any agency thereof. 


\title{
SHEAR BAND FORMATION IN PLASTIC BONDED EXPLOSIVE (PBX)
}

\author{
T. N. Dey and J. N. Johnson \\ Los Alamos National Laboratory, Los Alamos, NM 87545
}

\begin{abstract}
Adiabatic shear bands can be a source of ignition and lead to detonation. At low to moderate deformation rates, 10-1000 s-1, two other mechanisms can also give rise to shear bands. These mechanisms are: 1) softening caused by micro-cracking and 2) a constitutive response with a nonassociated flow rule as is observed in granular material such as soil. Brittle behavior at small strains and the granular nature of HMX suggest that PBX-9501 constitutive behavior may be similar to sand.
\end{abstract}

A constitutive model for the first of these mechanisms is studied in a series of calculations. This viscoelastic constitutive model for PBX-9501 softens via a statistical crack model. A sand model is used to provide a non-associated flow rule and detailed results will be reported elsewhere. Both models generate shear band formation at $1-2 \%$ strain at nominal strain rates at and below $1000 \mathrm{~s}^{-1}$. Shear band formation is suppressed at higher strain rates. Both mechanisms may accelerate the formation of adiabatic shear bands.

\section{INTRODUCTION}

Thermal softening as a cause of shear band formation in materials has been extensively studied. Such adiabatic shear bands in explosives may be a source of ignition and lead to detonation $(4,5,8,10)$. At low to moderate deformation rates, two other mechanisms can also give rise to shear bands, and require only a small strain to do so. By concentrating deformation, these mechanisms may accelerate the formation of adiabatic shear bands. The two mechanisms are mechanical softening caused by micro-cracking and a constitutive response characterized by a nonassociated flow rule.

In order to better understand the characteristics of these mechanisms, we have carried out a series of numerical calculations that simulate deformation of a plastic-bonded explosive (PBX) subjected to various loading conditions. In this paper we report results for calculations that use a constitutive model that generates a visco-elastic response together with mechanical softening due to micro-cracking. This model is intended to mimic the behavior of PBX9501.

\section{CONSTITUTIVE MODEL}

The constitutive model separates the material response into volumetric and deviatoric components. Because of the low pressure obtained in the simulations discussed in this paper, a volumetric response characterized by a constant bulk modulus is sufficient. The deviatoric response is divided into two components acting in series with each other.

One component is modeled as five Maxwell spring and dashpot elements acting in parallel to each other. This component produces a viscoelastic response. A Maxwell spring and dashpot element is the ideal behavior of a linear spring and a linear dashpot acting in series with each other. The spring is characterized by its contribution to the shear 
modulus of the material while the dashpot has characteristic relaxation time. For deformations occurring more rapidly than the characteristic time, the Maxwell element adds its stiffness to the other elements. For deformations on a time scale much longer than the characteristic time, the Maxwell element adds no stiffness.

In series with the viscoelastic component is a fracture mechanics component. Addessio and Johnson (1) developed this model as a simplification of a statistical crack mechanics model of Dienes(3). The model is characterized by a critical stress intensity factor, a mean crack size, an exponent that determines the crack growth velocity for conditions both below and above the critical stress intensity, and a maximum crack growth velocity. Model values are based on data from room temperature experiments on PBX-9501 by Gray et al. (7).

\section{CALCULATIONS}

In order to understand the general characteristics of shear band formation associated with this model, we performed a series of simple plane strain calculations each at a different nominal strain rate. The mesh was $0.01 \mathrm{~m}$ wide and $0.02 \mathrm{~m}$ high. Roller boundary conditions (no normal displacement but free tangential slip) were applied to the left and bottom edges. The right side was a free surface. The top surface was displaced vertically with no lateral constraint at constant velocity. The average vertical strain rate in each calculation was chosen from the

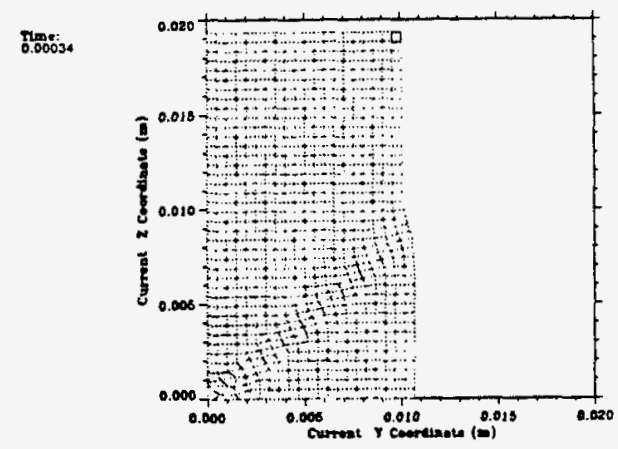

FIGURE 1. Deformed mesh showing shear band formed when average strain rate is $100 \mathrm{~s}^{-1}$. range of $10-10000 \mathrm{~s}^{-1}$. The meshes had 20 cells in the horizontal direction and 40 in the vertical direction. The time scale associated with the maximum crack growth velocity implies an internal length scale for this model and a finite width for any shear band that may form. The mesh used was found to resolve these shear bands adequately.

One cell at either the lower right or left of the mesh was given a slightly larger initial mean crack length causing it to be slightly weaker. Some form of perturbation like this is required to trigger shear band formation in the absence of loading inhomogeneity.

Figure 1 shows a mesh deformed at a nominal rate of $100 \mathrm{~s}^{-1}$. A shear band has clearly formed from the perturbation at the lower left corner of the sample. The width of the shear band near the perturbation is limited by the small size of the perturbation. Away from the perturbation, the shear band broadens to a width of five or so computational cells.

Figure 2 shows average vertical stress plotted as a function of average vertical strain for a number of different average strain rates. A stress drop is clearly visible in the curves for the lower strain rates. The beginning of this stress drop is coincident with the beginning of localization. The shear band is fully formed by the time the stress drop is complete. For strain rates of $1000 \mathrm{~s}^{-1}$ or less, the peak stress is reached at about $1 \%$ axial strain.

Figure 3 shows a plot of the mesh for a calculation with a nominal strain rate of $1000 \mathrm{~s}^{-1}$. It is clear from that no significant localization has

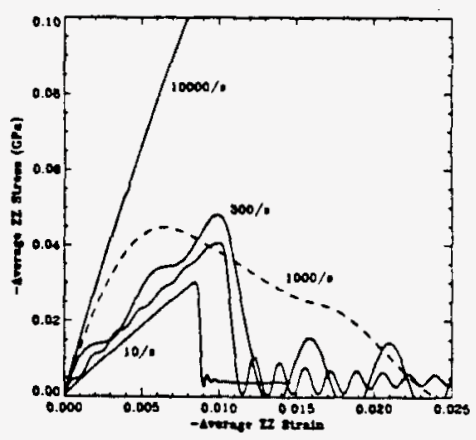

FIGURE 2. Average vertical stress vs. average vertical strain for each calculation. 
happened. The stress-strain curve in Figure 2 for this calculation shows a stress drop, but it is apparent that the drop is gradual. Evidently, inertial effects are now sufficiently large and the internal length scale for this material model is sufficiently long that there is not enough time for localization occur. At this and all higher strain rates, this model exhibits no tendency to generate shear bands.

\section{DISCUSSION}

A number of studies of shear band formation caused by thermal softening in metals indicate that strains must be substantial, $10-50 \%$, before shear bands form $(6,9,11)$. The numerical studies described here indicate that the mechanical softening in the viscoelastic microcracking model can lead to shear band formation at much smaller strains, only $1 \%$ strain in the examples shown here.

The shear band width generated by the model studied here is much greater than observed for the thermal softening mechanism. The numerical results give widths of a few $\mathrm{mm}$, while the thermal mechanism is associated with shear bands that are one to three orders of magnitude narrower. It is unlikely that the mechanism studied here is, by itself, able to generate sufficiently high temperatures to cause ignition of the explosive since the deformation is still being spread over a substantial volume due to the large shear band width.

The mechanism studied here may trigger

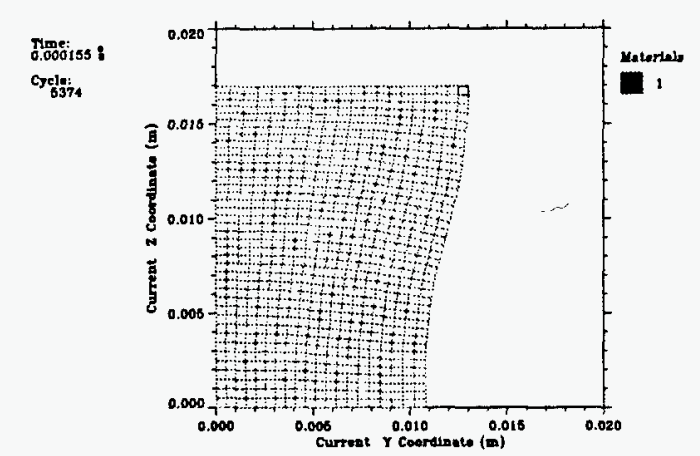

FIGURE 3. Deformed mesh indicating no shear band formation for strain rate of $1000 \mathrm{~s}^{-1}$. adiabatic shear band formation at much smaller average strains than those occurring if the thermal softening mechanism were acting alone. Even though the viscoelastic microcracking model by itself produces a shear band too wide to generate high temperature, locally it does produce high strain. These locally high strains, which are one to two orders of magnitude greater than the average strain, may activate the thermal softening mechanism. This, in turn, may lead to more localization and to high temperatures while the average strain is still only a few percent. The current viscoelastic microcracking model does not include any thermal softening effects; however, our research plans include examining this issue.

Localization and shear band formation was only observed at average strain rates less than $1000 \mathrm{~s}^{-1}$ for the model studied in this work. At sufficiently high strain rate in a metal, above $10^{5} \mathrm{~s}^{-1}$, Wright and Walter (11) noted that shear band formation was inhibited due to inertial effects. The shear bands observed to form in this work are about two orders of magnitude larger than those in (11). The ratio of critical strain rates is about the same, indicating results consistent with that previous study.

This low critical strain rate means that the softening mechanism studied here cannot contribute to shear band formation under shock conditions. In many accident scenarios, however, deformation rates are below $1000 \mathrm{~s}^{-1}$. Consequently, the mechanism discussed here may contribute to sensitivity in these cases.

We have performed similar studies on a model with a non-associated flow rule mechanism for generating localization, and found similar results. We have also performed some initial calculations of experiments described by Asay et al. (2) and found good qualitative agreement when either of these models is used, but not when only a thermal softening mechanism is used. These results will be reported as they become available.

\section{ACKNOWLEDGMENTS}

This worked was performed for the High Explosives Safety and Performance program of the U. S. Department of Energy. Los Alamos National 
Laboratory is operated by the Univ. of Californis for the U.S. Department of Energy.

\section{REFERENCES}

1. Addessio, F. L. and Johnson, J.N., J. Appl. Phys., 67, 3275 3286, (1990).

2. Asay, B. W., Henson, B. F. and Funk, D. J., "Direct Measurement of Strain Field Evolution During Dynamic Deformation of an Energetic Material," presented at the 1997 Topical Group on Shock Compression Meeting, Amherst, MA, 27 July-1 August 1997.

3. Dienes, J. K., Mech. Mater., 4, 325 (1985)

4. Field, J.E., Swallowe, G. M. and Heavens, S.N, Proc. R. Soc. Lond. A 382, 36-42 (1982).

5. Frey, R.B., in Proceedings of the Seventh Symposium on Detonation, Office of Naval Research, Arlington, VA, 1981. pp. $36-42$.

6. Grady, D. E. and Kipp, M. E., J. Mech. Phys. Solids, 35, $95-$ 118 (1987).

7. Gray III, G.T. .. Blumenthal, W.R,. Cady, C.M. and Idar, D.J, "Influence of Temperature on the High-Strain-Rate Mechanical Behavior of PBX 9501 and PBXN-9," presented at the 1997 Topical Group on Shock Compression Meeting, Amherst, MA, 27 July-1 August 1997.

8. Kerrisk, J.F., Los Alamos National Laboratory Report LA. 13127 (1996).

9. Molinari, A. and Clifton, R. J., J. Appl. Mech., 54, 806-812 (1987).

10. Winter, R. E. and Field, J.E., Proc. R. Soc. Lond. A 343, 299 413 (1975).

11. Wright, T. W. and Walter, J. W.; J. Mech. Phys. Solids, 35, $701-720$ (1987). 
M98000627

||||||||||||||||||||.

Report Number (14)LA-UR,-97-2857 CONF-970707--

Publ. Date (11) 199710

Sponsor Code (18) $\frac{D O E / D P, X F}{U C-741, D O E / E R}$
UC Category (19)

DOE 